\title{
Research
}

\section{Climate-driven shifts in the distribution of koala-browse species from the Last Interglacial to the near future}

\author{
Farzin Shabani, Mohsen Ahmadi, Katharina J. Peters, Simon Haberle, Antoine Champreux, Frédérik Saltré \\ and Corey J. A. Bradshaw
}

F. Shabani (https://orcid.org/0000-0002-5100-8921) ■ (farzin.shabani@flinders.edu.au), K. J. Peters (https://orcid.org/0000-0002-5967-0928), A. Champreux (https://orcid.org/0000-0001-9910-2223), F. Saltré (https://orcid.org/0000-0002-5040-3911) and C. J. A. Bradshaw (https://orcid. org/0000-0002-5328-7741), ARC Centre of Excellence for Australian Biodiversity and Heritage, Global Ecology, College of Science and Engineering, Flinders Univ., Adelaide, SA, Australia. - M. Ahmadi (https://orcid.org/0000-0002-9657-699X), Dept of Natural Resources, Isfahan Univ. of Technology, Isfahan, Iran, and Swiss Federal Research Inst. WSL, Dynamic Macroecology Group, Birmensdorf, Switzerland. - S. Haberle (https:/lorcid.org/0000-00015802-6535), Dept of Archaeology and Natural History, College of Asia and the Pacific, The Australian National Univ., Acton, ACT, Australia.

\section{Ecography}

42: 1587-1599, 2019

doi: $10.1111 /$ ecog. 04530

Subject Editor: Jessica Blois Editor-in-Chief: Miguel Araújo Accepted 20 May 2019
The koala's Phascolarctos cinereus distribution is currently restricted to eastern and south-eastern Australia. However, fossil records dating from $70 \pm 4 \mathrm{ka}\left(\mathrm{ka}=10^{3} \mathrm{yr}\right)$ from south-western Australia and the Nullarbor Plain are evidence of subpopulation extinctions in the southwest at least after the Last Interglacial $(\sim 128-116 \mathrm{ka})$. We hypothesize that koala sub-population extinctions resulted from the eastward retraction of the koala's main browse species in response to unsuitable climatic conditions. We further posit a general reduction in the distribution of main koala-browse trees in the near future in response climate change. We modelled 60 koala-browse species and constructed a set of correlative species distribution models for five time periods: Last Interglacial $(\sim 128-116 \mathrm{ka})$, Last Glacial Maximum ( 23-19 ka), Mid-Holocene ( 7-5 ka), present (interpolations of observed data, representative of 1960-1990), and 2070. We based our projections on five hindcasts and one forecast of climatic variables extracted from WorldClim based on two general circulation models (considering the most pessimistic scenario of high greenhousegas emissions) and topsoil clay fraction. We used 17 dates of koala fossil specimens identified as reliable from $70( \pm 4)$ to $535( \pm 49) \mathrm{ka}$, with the last appearance of koalas at $70 \mathrm{ka}$ in the southwest. The main simulated koala-browse species were at their greatest modelled extent of suitability during the Last Glacial Maximum, with the greatest loss of koala habitat occurring between the Mid-Holocene and the present. We predict a similar habitat loss between the present and 2070. The spatial patterns of habitat change support our hypothesis that koala extinctions in the southwest, Nullarbor Plain and central South Australia resulted from the eastward retraction of the dominant koala-browse species in response to long-term climate changes. Future climate patterns will likely increase the extinction risk of koalas in their remaining eastern ranges.

Keywords: climate change, extinctions, fossil age reliability, fossil distribution, koala, species distribution models 


\section{Introduction}

The predicted impacts of anthropogenic climate disruption on global biodiversity include shifts in the ranges of species and the acceleration of extinctions (Parmesan and Yohe 2003, Bellard et al. 2012, Urban 2015, Strona and Bradshaw 2018). Within a species' range, the changes in leading- and trailing-edge populations are potentially different (Thuiller et al. 2008, Anderson et al. 2009, Saltré et al. 2015b, Shabani et al. 2017). For example, the emerging localities of greater suitability can induce leading-edge migration, while trailing-edge habitats experience fragmentation and heightened extinction risk (Parmesan and Yohe 2003, Pearson et al. 2009, Thomas 2010). However, despite many potential behavioural, physiological and evolutionary adaptations (e.g. adaptation to different browse species, or physiological shift of thermal tolerances) and range shifts occurring, these will not be sufficient to offset overall population declines in many species, because rapid, accumulated changes in ecosystems (e.g. habitat loss, water and air pollution, climate change, ultraviolet light exposure, invasive species, disease) generally outpace adaptation (Parmesan 2006).

The marsupial koala Phascolarctos cinereus is one widespread Australian species (Fig. 1 top panel) that is hypothesized to have had substantial range contractions during the Last Glacial Maximum, and is also predicted to continue to contract over the next several decades (Gordon et al. 1988, Adams-Hosking et al. 2011a, b, Lunney et al. 2012). Climate change has been hypothesized as the possible driver of observed territorial shifts in koalas (Reckless et al. 2018) by promoting physiological stress, and a greater risk of chlamydiosis outbreaks and other diseases (i.e. bacterial infection compromising health) (Seabrook et al. 2014, Reckless et al. 2018). The species faces additional challenges from several other stressors, including habitat fragmentation, land clearing, dog attacks and road kill (McAlpine et al. 2015). In fact, just over two centuries after Europeans first saw koalas, this species is now classified as threatened across two-thirds of its range, with $>50 \%$ population declines (Melzer et al. 2000), although there are small populations apparently not experiencing recent declines (Lunney et al. 2016). The species is listed as Vulnerable in Queensland, New South Wales and the Australian Capital Territory by the IUCN Red List and the Australian Environment Protection and Biodiversity Conservation Act 1999 (Sequeira et al. 2014). Adams-Hosking et al. (2016) recently applied expert elicitation to provide a current total estimated size of the koala population between 144000 and 605 000, with an estimated average decline of $24 \%$ over the past three generations. Estimated percentages of losses in Queensland, New South Wales, Victoria and South Australia were 53, 26, 14 and 3\%, respectively. Lunney et al. (2014) also documented a long-term shrinkage in the distribution of koalas across the Eden region $\left(\sim 7000 \mathrm{~km}^{2}\right)$ in south-eastern New South Wales due to climate change, particularly from drought and rising temperatures.
Koalas depend predominantly on eucalypts (mainly, Eucalyptus spp.) for food and shelter; therefore, the risk of koala populations going locally extinct depends ultimately on the amount of eucalypt forest cover (as well as housing density in the modern context) (Santika et al. 2014). As further evidence that threats to koalas are likely to increase, there are many studies that have examined the impact of climate change on different eucalypt species - the overall conclusion is that there are substantial changes expected in the distribution of different tree flora in Australia in the near future (Hughes et al. 1996, Hughes 2003, Austin and Van Niel 2011, Mok et al. 2012, Booth 2013, 2017b, Butt et al. 2013, Matusick et al. 2013, Booth et al. 2015). Other studies have documented that the land use-driven loss and fragmentation of forest habitats in Australia also threaten forest-dependent fauna (McGarigal and McComb 1995, McAlpine and Eyre 2002, McAlpine et al. 2006).

But changes in koala distribution have been occurring for much longer than since humans first arrived in Australia (i.e. sometime around $63.7-47.3 \mathrm{ka}$ ago, Saltré et al. 2016), with fossil records of koalas reliably dated (Rodríguez-Rey et al. 2016) back to over $150 \mathrm{ka}$ in south-western Australia, as well as specimens found in the Nullarbor Plain and on Kangaroo Island (Fig. 2). These observations lead to the hypothesis that the koalas of western and southern Australia dwindled to extinction because of decreasing habitat availability as the forests including their main browse species contracted in response to large climate fluctuations, at least since the Last Interglacial period ( 128-116 ka; Stirling et al. 1998). Because of insufficient reliable palaeodata for the koala (i.e. estimated fossil age uncertainty category A in Fig. 2, top left panel), it would be difficult to build and validate simulated palaeodistributions against independent data (Svenning et al. 2011), but it is possible to do this with various eucalypt species (see Results and Supplementary material Appendix 1 for the present-day koala distribution). By inferring the temporal shifts in the ranges of the most consumed browse species from palaeoclimate reconstructions using WorldClim, we can indirectly test whether changes in forest extent since the Last Interglacial (128-116 ka, Stirling et al. 1998) were likely responsible for local extirpations of forest-dependent koalas.

Our objectives in this paper were to: 1) investigate whether climate conditions during the Last Interglacial (128-116ka, Stirling et al. 1998), the Last Glacial Maximum ( 23-19 ka, Clark et al. 2009), the MidHolocene ( 7-5 ka; Steig 1999), and today can explain the spatio-temporal range shifts suggested by existing fossil koala specimens; 2) using an ensemble-hindcasting approach, examine specifically when and where the ranges of suitable koala-browse tree species would have isolated koala populations to the west of their current range using the same modelling approach, and 3) project the continental suitability of the main koala-browse species [based on contemporary occurrences] to 2070 assuming the most pessimistic scenario of the Intergovernmental Panel on 


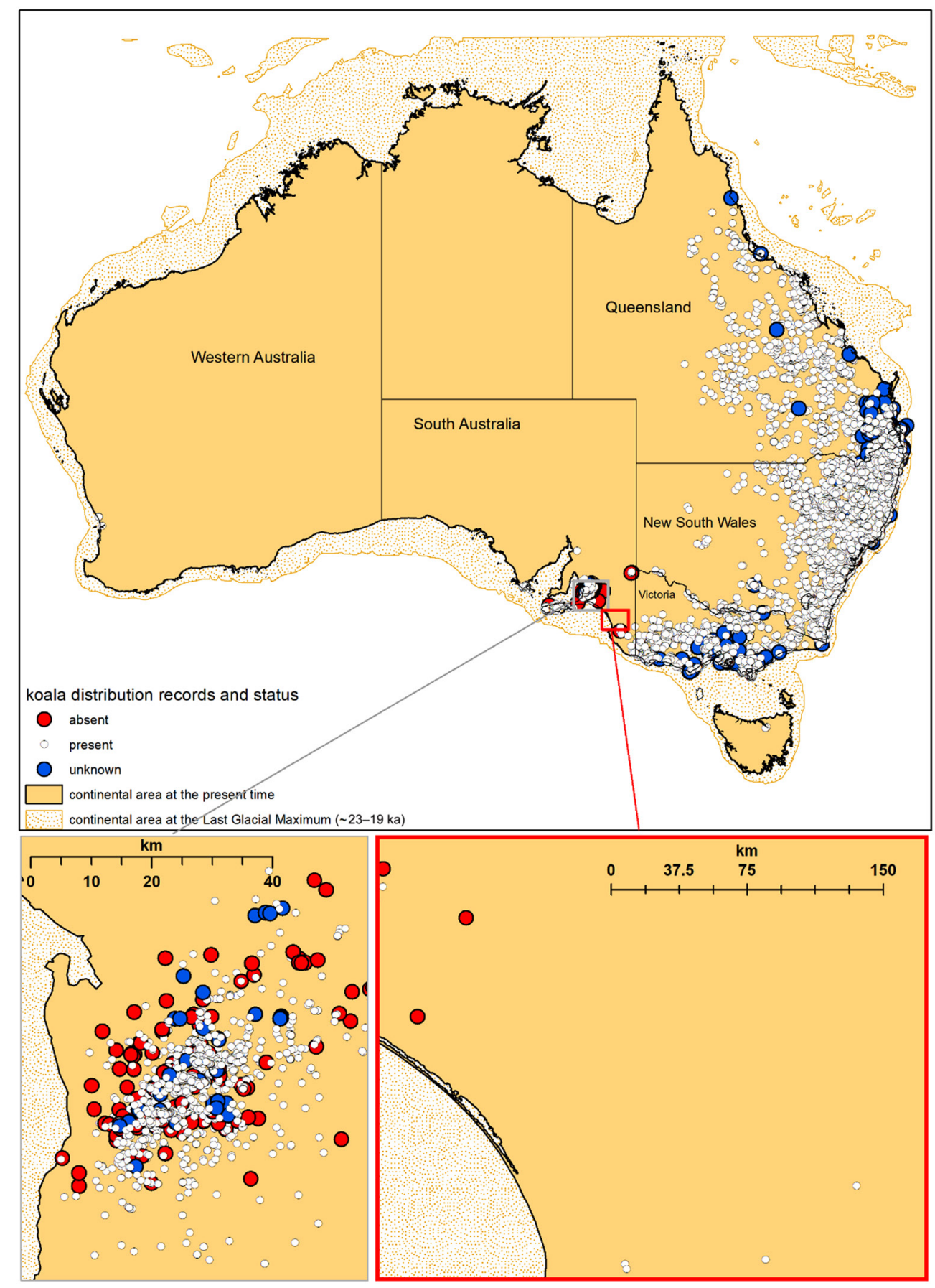

Figure 1. Current distribution of koalas based on data from the Atlas of Living Australia (ala.org.au), and continental area between today and the Last Glacial Maximum. Absence points $(n=142)$ and unknown $(n=443)$ are based on Atlas of Living Australia data for koala 'absent' and 'unknown occurrence status' records.

Climate Change's Representative Concentration Pathway (RCP 8.5 scenario of high greenhouse-gas emissions).

\section{Methods}

We developed ensemble species distribution models for koala-browse species based on contemporary koala-browse occurrences, climate, and non-climatic variables, and validated the models with contemporary koala-browse and koala occurrence records. We then projected these ensembles to three periods in the past back to the Last Interglacial, and one future time period (2070). We evaluated the performance of our past projections using fossil koala and fossil pollen data. 


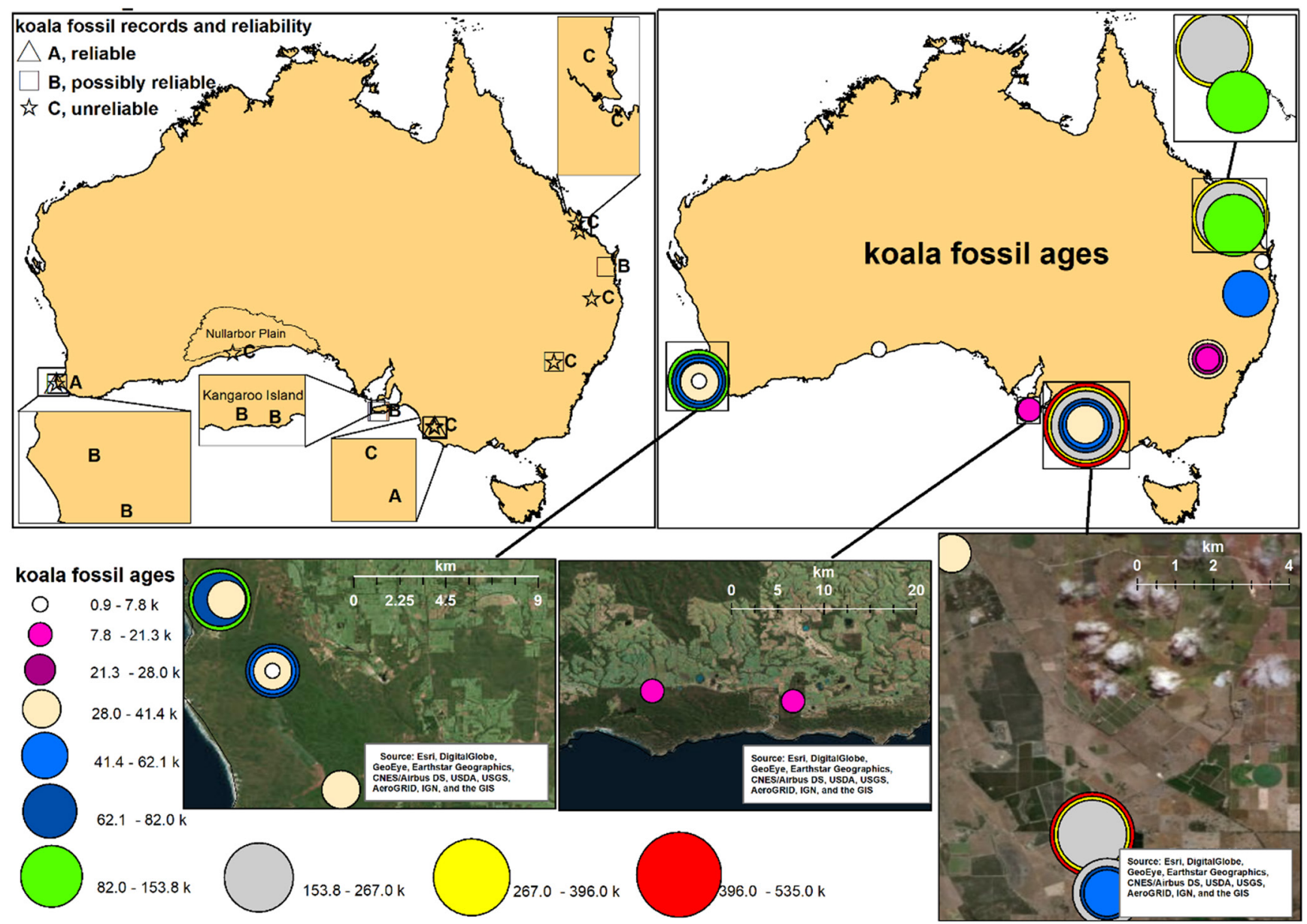

Figure 2. Spatial distribution of koala fossils and the reliability category of fossil ages for each record (derived from the 'FosSahul' database; Rodríguez-Rey et al. 2016). The change in land mass between the present and the Last Glacial Maximum is not shown (Fig. 1). In our study, we used koala fossil records merely as evidence of koala presence at a given location, so the age ranges given are purely informative.

\section{Contemporary/fossil data, and climate simulations}

Koalas tend to maximize their water intake by favouring trees with high leaf moisture, which varies among species and local water availability (Ellis et al. 2010, Wu et al. 2012, Davies et al. 2014). There is a considerable spatial variation in the consumption of different tree species by koalas across their current range (Phillips et al. 2000); for example, Eucalyptus robusta (swamp mahogany) and E. parramattensis (Paramatta red gum) are most selected by koalas in the coastal Port Stephens area of New South Wales (possibly due to the limitation of these species' spatial distributions). Similarly, koalas can live in a wide variety of habitats across Australia, mainly dominated by 72 different eucalypt species used by koalas as possible browse and shelter trees (Bryan 1997). However, biases have been recognized in past estimates of the koala's diet, so we used the recent list of likely browse species constructed from expert consultation and compiled by the New South Wales State Environmental Planning Policy for koala habitat protection (NSW Dept of Planning and Environment 2016) - this list includes 60 species for which sufficient Atlas of Living Australia contemporary distributional data exist to construct species distribution models (Supplementary material Appendix 1 Table A1).

The 'FosSahul' database (Rodríguez-Rey et al. 2016) currently lists over 9000 Australasian fossil records from 363 deposits, covering 478 species within 215 genera, with 27 representing extinct or extant megafauna (2559 records). The database contains 42 Phascolarctos-level records (after quality rating - see relevant procedure below); however, examining all known Web of Knowledge ${ }^{\ominus}$ published papers, published databases and personal communications from 2015 up to April 2018, we found an additional 10 valid records (after quality rating) after eliminating duplicate records and unverified information, and contacting authors and other experts as part of the record-validation process. Fossils can be dated using a range of methods, and the reliability of the age can vary widely depending on the dated remains and the type of dating technique used. Confidence in fossil ages is crucial when using these records to infer species' distributions, extinction dynamics, and the like (Saltré et al. 2015a). To assess 
the reliability of the associated fossil dates (quality rating) for each additional record, we followed a two-step protocol to provide an objective assessment of age reliability according to Rodríguez-Rey et al. (2015). In the first step, we assessed the reliability of a given age as a function of the dating procedure used, and in the second, we evaluated whether the association with the vertebrate fossil was based on an accurate scientific analysis of the physical surroundings from which it was extracted. Our quality ranking of the dates was based on three classes of reliability (A: reliable; B: possibly reliable; and $\mathrm{C}$ : unreliable). A detailed discussion on this methodology and associated criteria can be found in Rodríguez-Rey et al. (2015). See Supplementary material Appendix 1 Table A2 for the Phascolarctos fossil specimen dates and quality-rating outputs.

We used koala-browse species records, climate and non-climate variables for model simulations. We obtained contemporary distribution records of the 60 koala-browse species, and for koalas, from the Atlas of Living Australia (2018) (ala.org.au). For the 60 koala-browse species, we obtained 395583 distribution records from which we excluded records that were 1) without coordinate precision, 2) duplicate records, or 3) suspected outliers (i.e. coordinates out of the species' range). We then included one presence/ absence at each grid cell (even when there was $\geq 1$ record in a given grid cell) because the models we used for each cell demand only a presence or absence, and not a relative abundance (Shabani et al. 2016). We used the 'sp' package in $\mathrm{R}$ ver. 3.4.4 ( $\mathrm{R}$ Core Team) for processing all georeferenced occurrence data. We ended up with 36384 distribution records for the 60 koala-browse species. For koalas, we obtained 60476 records from which we similarly excluded records that were 1) without coordinate precision, 2) duplicate records, or 3) suspected outliers. We ended up with 58427 koala presences, 142 absences and 443 'unknowns' (Fig. 1). Thus, the total number of presence records for the koala-browse species and koalas together was 94811 .

An essential assumption of frequentist statistical methods is that recorded data are independent (i.e. randomly allocated samples with independent distributions), requiring that the entire area of interest has been randomly or systematically sampled. In practice, available data of the species' locations are spatially biased toward areas more easily assessed and/or better surveyed (Kramer-Schadt et al. 2013). This phenomenon is even more likely for data in global datasets (e.g. Global Biodiversity Information Facility or Atlas of Living Australia) originating from different sources with varying magnitudes and extents of field sampling (Wisz et al. 2008). Although some methods can cope better with spatially biased data than others (e.g. MaxEnt), spatial autocorrelation among locations can still result in biased parameter estimates and over-representation of some regions (Dormann et al. 2007). We applied two strategies to reduce the influence of potential biases in the occurrence points of the species we modelled: spatial filtering (Kramer-Schadt et al. 2013) and background weighting (Elith et al. 2010). For spatial filtering, we removed all the repeated points within a 5-km buffer radius. Because selecting pseudo-absences can randomly bias model predictions (Sequeira et al. 2012, Renner and Warton 2013), we used background weighting to remove some of the potential biases. Here, we provided models with environmental data as spatially biased as the occurrence data. For each koala-browse species, we calculated the kernel density surface of a species' occurrence points using 10000 background points to derive the probability distribution of the density surface. We thus simulated species-specific 'bias files' representing the relative sampling effort for each koala-browse species.

\section{Climate/soil data, and continental area}

We sourced climate variables as distribution predictors for the five time periods (Last Interglacial, Last Glacial Maximum, Mid-Holocene, present, and 2070) from the WorldClim database (worldclim.org), at a resolution of $2.5 \mathrm{~min}(4.5 \times 4.5 \mathrm{~km})$. Nineteen WorldClim climatic variables are highly correlated, so to remove multi-collinearity we calculated the variance inflation factor for each of the climatic variables. Before modelling, we used the 'usdm' package (Naimi 2015) to calculate the variance inflation factor (Guisan et al. 2017) and set a correlation threshold of 0.7 to detect multicollinearity among explanatory variables to remove the redundant ones (Ahmadi et al. 2017). We eventually selected six variables as plausible distribution predictors: 1) bio1 (annual mean temperature in ${ }^{\circ} \mathrm{C}$ ); 2) bio4 (temperature seasonality $=$ standard deviation $\times 100) ; 3$ ) bio5 (maximum temperature of the warmest month in ${ }^{\circ} \mathrm{C}$ ); 4) bio12 (annual precipitation in $\mathrm{mm}$ ); 5) bio14 (precipitation of the driest month in $\mathrm{mm}$ ); and 6) bio19 (precipitation of the coldest quarter). We used these climate variables to predict and project the distribution of the species at a coarse geographic scale to cover annual, seasonal and absolute characteristics of climate that could dictate the growing conditions experienced by trees.

The WorldClim database provides 19 global circulation models for the future, 9 for the Mid-Holocene and 3 for the Last Glacial Maximum. We selected two of these - the Community Climate System Model (CCSM4; <www. cesm.ucar.edu/models/ccsm4.0>) and the Model for Interdisciplinary Research on Climate-Earth Systems Model (MIROC-ESM; Watanabe et al. 2011) that were consistently available for most of the time periods we considered (2070, Mid-Holocene and Last Glacial Maximum). The use of multiple, global circulation models results in better projection accuracy while maintaining reliability compared to using only one (Schepen and Wang 2013).

We used the Australian topsoil clay fraction (\% weight) sourced from the Harmonized World Soil Database ver. 1.2 (Nachtergaele et al. 2009) at a resolution of $2.5 \mathrm{~min}$. Soil texture and its components are determined by particle size, such that soils containing a high concentration of clay particles enhance water and mineral retention available for plant growth. The topsoil clay fraction therefore provides 
a reasonable approximation of soil and nutrient availability to plants (Duursma et al. 2013), especially considering that Australia is characterized by a prevalence of highly leached, nutrient-poor soils (Duursma et al. 2013) and dry conditions. We assumed that soil conditions were identical in all five periods mainly because these tree species are unlikely to shift as quickly as climate conditions, and because of the lack of any palaeosoil distribution data at the continental scale (Booth 2017a).

During the Last Glacial Maximum, Tasmania was connected to mainland Australia, and the coastal regions of Australia exceeded those of the current time given lower sea levels. The continental area estimation during the Last Glacial Maximum is from WorldClim palaeoclimate dataset from CCSM4 model ( $120 \mathrm{~m}$ below present sea level) (COHMAP Members 1988). However, the Last Interglacial, Mid-Holocene and 2070 all had or will have continental extents approximately equivalent to today's coastline (Supplementary material Appendix 1 Fig. A1). Hence, our downscaled WorldClim palaeoclimate dataset includes the greater continental area of Australia during the Last Glacial Maximum. Additionally, due to the absence of topsoil clay fraction data for the continental area of Australia during the Last Glacial Maximum, we excluded this parameter in the distribution modelling pertaining to that period.

\section{Species distribution modelling}

We applied the 'biomod2' package ensemble platform for species distribution modelling (Thuiller et al. 2009) in the $R$ environment ( $\mathrm{R}$ Core Team) to predict suitable koala habitats in terms of the distributions of individual koala-browse species. The method processes several modelling techniques simultaneously to create a consensus, or 'ensemble', model (Araújo and New 2007, Thuiller et al. 2009). We selected four techniques for the ensemble predictions: generalized linear model, maximum entropy (MaxEnt), generalized boosting model, and surface range envelope (also known as 'Bioclim' - refer to Booth et al. (2014) for information on early developments and initial applications), to form an integrated prediction of the 60 koala-browse species (contemporary occurrences) we identified (see Results), with thirty replicates each. Our aim was to produce predictions of relative browse-species suitability, rather than attempt to predict the real distributions of these species. Finally, we calculated the ensemble predictions using the evaluation statistic-weighted average of each model (Thuiller et al. 2009).

In structuring the final distribution models, we established the areas of greatest suitability for each of the 60 koalabrowse species to construct a 'core' habitat for koalas in each of the projected time periods. Thereafter, we compared these predicted core habitats with the current suitable habitats to identify the extent of habitat change over different time periods (past and future) based on a quantile classification technique. For our analysis, the quantile method suited the classification of the extent of habitat loss and gain relative to the present. The method is effective for the classification of areas, moving equal quantities of pixels into each group without separation difficulties (Shabani et al. 2018). There are four main quantile methods (natural breaks, equal intervals, geometrical intervals and standard deviations) available for classifying scaled data (Ayalew et al. 2004, Ayalew and Yamagishi 2005, Papadopoulou-Vrynioti et al. 2013, Tehrany et al. 2017), with the appropriate method depending on the type of data and their application. A disadvantage of the equal-intervals approach is that it can place values of extreme difference into the same class, or similar values in adjacent classes, which are best minimized by increasing the number of classes. Alternatively, natural breaks and standard deviation structure the data into non-user-defined categories. Natural breaks find the most suitable natural categories inherent in the data, while standard deviation groups are based on deviations from mean values. In certain analyses of species distributions where the goal is to highlight large discontinuities in the distribution of suitability scores, natural breaks tends to be the most appropriate choice (Xiao et al. 2006). We used the Atlas of Living Australia (2018) to compile the eucalypt distribution dataset. Combining the standard average projections of all 60 species as a mean suitability per projected pixel enabled us to compare results with independent palaeoarchives (i.e. pollen records; see Discussion and Supplementary material Appendix 1).

\section{Model performance}

We used $25 \%$ of the koala-browse species records together with koala data for the evaluation of model performance (see Results). We used the area under the (receiver operating characteristic) curve (AUC) and the true skill statistic (TSS) to evaluate model performance, based on the known capacity of these statistics to respond to prevalence in species distribution data (Swets 1988, Allouche et al. 2006). Using the BIOMOD framework, we estimated the individual contribution of all variables in the species distribution models and evaluated the responses of the species distributions to the gradients of explanatory variables based on the response curves derived from each model.

The area under the curve is a reliable measure of discrimination ability, but when estimations are based only on presence data, it has limitations for ecological realism in the modelled distributions (Lobo et al. 2008, JiménezValverde 2014, Booth 2017c). We therefore also used the true skill statistic to assess model performance because this metric is independent of prevalence. Allouche et al. (2006) demonstrated that the true skill statistic represents an intuitive method to assess the predictive performance of species distribution models transposed into presence-absence mapping.

From the relative suitability map (Guillera-Arroita et al. 2014), we determined a threshold below which we considered the species 'absent'. This threshold method is commonly used to transform continuous probabilities of presence to presence/absence data in species distribution models (Nenzén and Araújo 2011). The 10th-and 5 th-percentile training presences 
are two thresholds commonly used in ecology (Pearson et al. 2002). Other possible methods include establishing a specific sensitivity or specificity threshold (Cantor et al. 1999), maximizing sensitivity plus specificity, maximizing kappa (which includes both sensitivity and specificity), maximizing the percent of points correctly classified, and maximizing the agreement of predicted and observed distributions (Guisan et al. 1998). We initially calculated the 10th percentile of the suitability score at presence points, but in expanding the analysis, we also tested the 5th-percentile threshold, as well as the maximum sensitivity plus specificity to assess the performance. Based on the number of pixels gaining or losing habitat suitability in the climatic projections, we then measured range shifts of the koala-browse species over the time periods we considered.

\section{Data deposition}

Data available from the Dryad Digital Repository: <https:// doi.org/10.5061/dryad.r79k7sc> (Shabani et al. 2019).

\section{Results}

\section{Model performance and spatial outputs}

According to the area under the curve and the true skill statistic, all four species distribution models performed well overall for the 60 koala-browse species (AUC $>0.88$ and TSS > 0.71) (Fig. 3, refer to Supplementary material Appendix 1 Table A3 for the performance of the discrimination capacity and accuracy of different algorithms for each species, and Supplementary material Appendix 1 Table A4 for climate variable contributions for each eucalypt species). The averaged ensemble-model suitability maps (Fig. 4) for

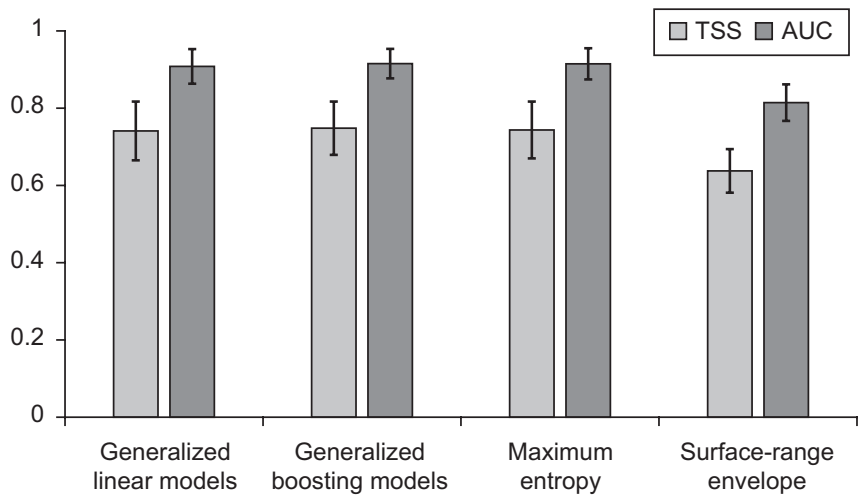

Figure 3. Overall performance of the discrimination capacity and accuracy of different algorithms to predict 60 koala-browse species (based on contemporary occurrences). AUC =area under the (receiver operating characteristic) curve that assesses the accuracy of ordinal-score models. TSS (true skill statistic) is an intuitive method of performance measurement for species distribution models (refer to Supplementary material Appendix 1 Table A3 for the performance of the discrimination capacity and accuracy of different algorithms to predict each koala-browse species).
60 species over the five periods (Last Interglacial period 128-116ka, Last Glacial Maximum 23-19ka, MidHolocene $\sim 7-5 \mathrm{ka}$, the present and 2070) are presented along with their medians and mean deviations of suitability derived over all 60 species (Supplementary material Appendix 1 Fig. A2).

Individual model (generalized linear model, maximum entropy, generalized boosting model and surface-range envelope [Bioclim]) rankings of variable importance varied among species and approaches. For example, generalized linear models identified maximum temperature of the warmest month (bio5) as the most important predictor for E. robusta, while the generalized boosting model identified total precipitation of the driest month (bio14) for the same species. Additionally, the average importance of the variables across species varied. For example, maximum temperature of the warmest month and total precipitation of the driest month were the strongest predictors for E. robusta.

The median ensemble habitat suitability across all contemporary 60 koala-browse species reached a relative maximum habitat suitability sometime between the Last Glacial Maximum and the Mid-Holocene (Fig. 4 and Supplementary material Appendix 1 Fig. A2), although there was considerable spatial variation in the areas of loss and gain relative to the current suitability across the continent (Fig. 4, 5, Supplementary material Appendix 1 Fig. A2, Table A5), demonstrating that forest composition was likely highly variable in terms of actual species presence during these times and places in particular. The mean standard deviation of suitable habitat over all 60 species for all individual pixels covering the continent (Fig. 4, bottom row) indicates high variation in suitability among species. For instance, from the Last Glacial Maximum to the Mid-Holocene and for the forecasts to 2070, E. vicina, E. albnes and E. camphora had relative range losses. Similarly, there were also substantial losses of habitat suitability in the south-western and southern regions for E. bicostata, E. camphora, E. cinerea, E. dalrympleana, E. goniocalyx, E. largiflorens, E. microcarpa, E. parramattensis, E. pauciflora, E. pseudoglobulus, E. radiata, E. globulus, E. microcorys, E. ovata and E. viminalis from the Last Glacial Maximum to the Mid-Holocene (Supplementary material Appendix 1 Fig. A2). The ensemble models projected a complete loss of suitable habitat for E. globulus, E. pauciflora, E. macrorhyncha, E. cypellocarpa and E. cinerea in the southwestern and southern regions by 2070 (Supplementary material Appendix 1 Fig. A2). We emphasize that we used koala fossil records merely as evidence of koala presences at given locations, and that the displayed age range (Fig. 2) is purely informative; the location of koala fossils records should be interpreted in combination with the results derived from the koala-browse species.

We compared the overall extent of habitat gain and loss for the 60 koala-browse species between all modelled temporal windows and the present (Fig. 5; see habitat gain and loss for individual species in Supplementary material Appendix 1 Table A5). Suitable habitat was $71 \%$ less widespread during the Last Interglacial compared to present day (Fig. 5), but we projected it to lose up to $62 \%$ of its current range by 2070 . 

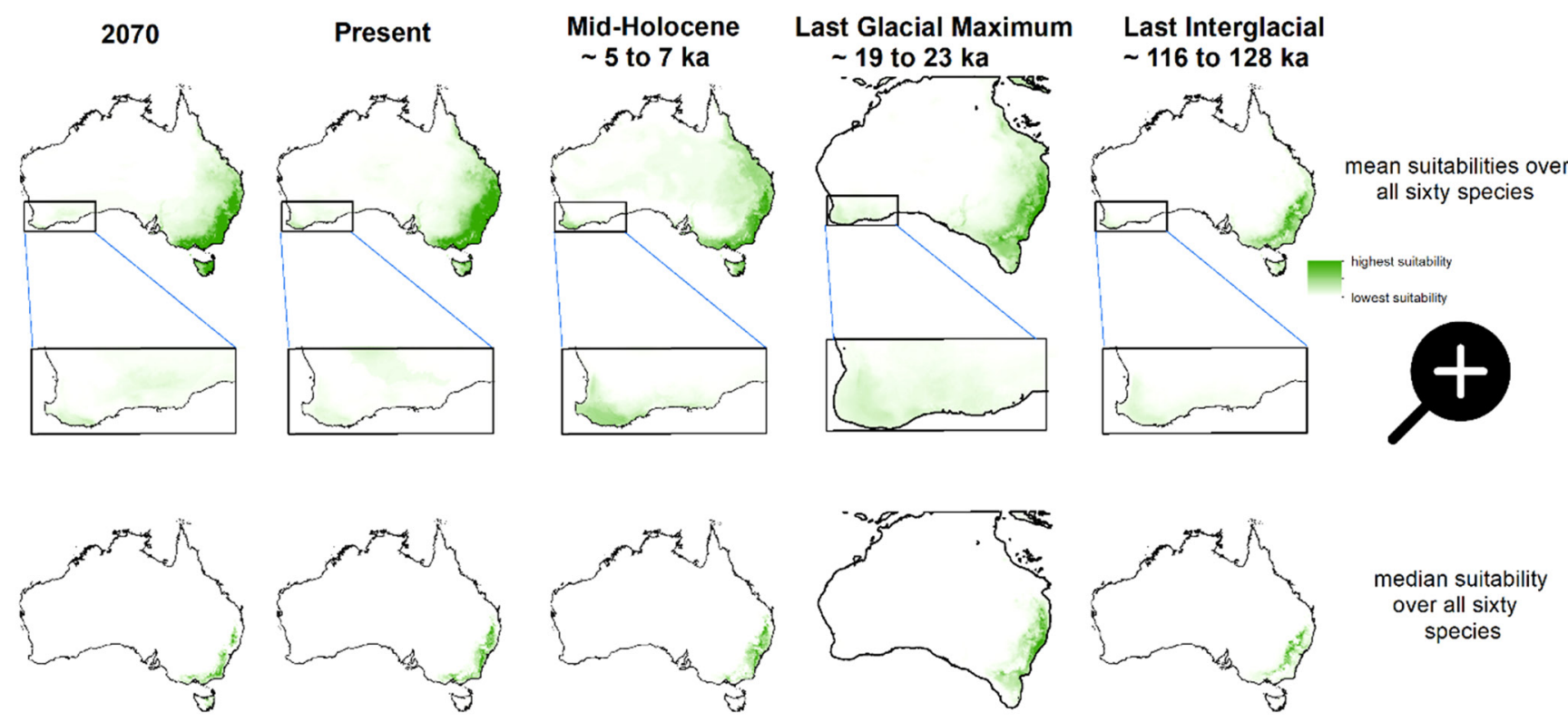
median suitability over all sixty species
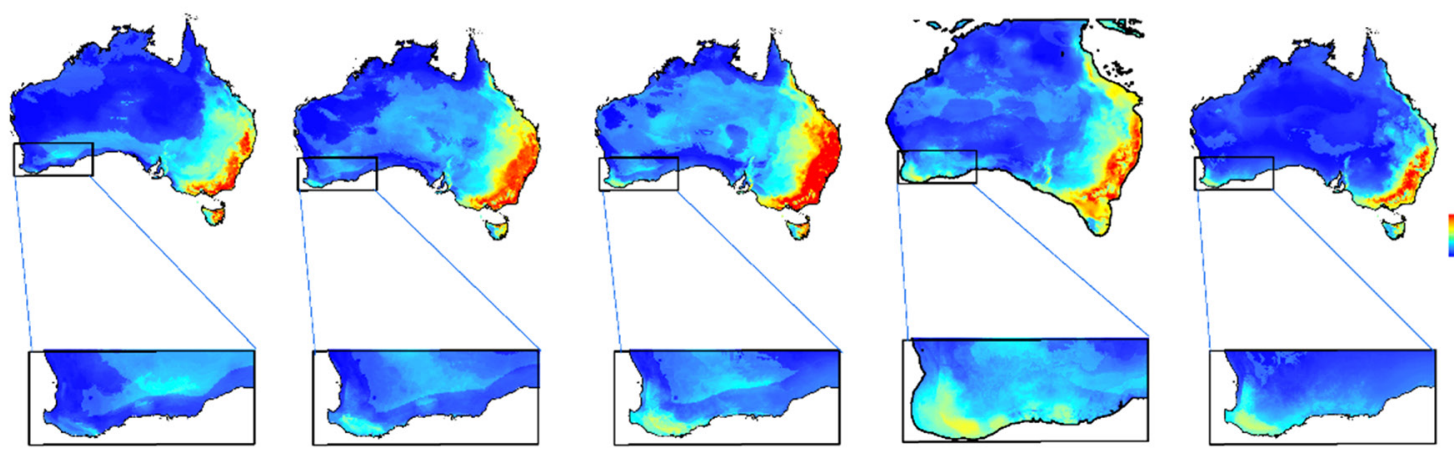

standard deviation over all sixty species

Figure 4. Ensemble model outputs (two global circulation models: CCSM4 and MIROC-ESM) and four modelling techniques (generalized linear model, maximum entropy, generalized boosting model and surface-range envelope) showing the mean, median and the standard deviation of niche suitabilities for 60 koala-browse species for the five time periods examined (Last Interglacial 128-116ka, Last Glacial Maximum 23-19ka, Mid-Holocene $7-5 \mathrm{ka}$, the present, and 2070). Dark green indicates the highest suitability and light green indicates the lowest. White indicates unsuitability (refer to Supplementary material Appendix 1 Fig. A2 for current distribution records and ensemble model outputs for each species). The centre panels indicate the median suitability over all 60 species per grid cell and the mean deviation of these per cell (bottom panel) indicate the overall response and inter-species variability in predicted responses for the entire continent.

The combined distribution of all 60 species is projected to shift $22 \%$ of their suitable present day-habitat to new regions (i.e. range gain) by 2070 . Our projections of the current suitable climatic range of koala-browse species compared to the Last Glacial Maximum indicate a reduction (60\%) in the availability of suitable ranges (i.e. range loss) for most species (Fig. 5, Supplementary material Appendix 1 Fig. A2, Table A5).

The mean suitability of the 60 koala-browse species matches well with the koala's projected present-time habitat distribution (almost 99\% of the koala's present distribution falls within suitable browse-species habitat categories) (Supplementary material Appendix 1 Fig. A3); for example, New South Wales has the highest koala suitability (0.84), followed by Victoria (0.81), South Australia (0.79), Queensland (0.78), Tasmania (0.30) and then Western Australia (0.10). These results can be considered a type of additional model validation - the current spatial patterns of koala habitat thus support our hypothesis that koala extinctions in the southwest, Nullarbor Plain and central South Australia resulted from the eastward retraction of forests, characterized by the loss of species like E. bicostata, E. cinerea, E. globulus and E. goniocalyx in response to long-term climate changes (Supplementary material Appendix 1 Fig. A3). The spatial comparison of these general results to available palaeo-vegetation records (Supplementary material Appendix 1 Fig. A5, Table A6 and Discussion) shows that despite pollen records not validating the decrease in Eucalyptus suitability between the Mid-Holocene and the present in south-eastern Australia, our results agree with the increase in Eucalyptus suitability between the Last Glacial Maximum and the present in the same region, and a slight increase in Eucalyptus suitability in south-western Tasmania from the Mid-Holocene to the present. 


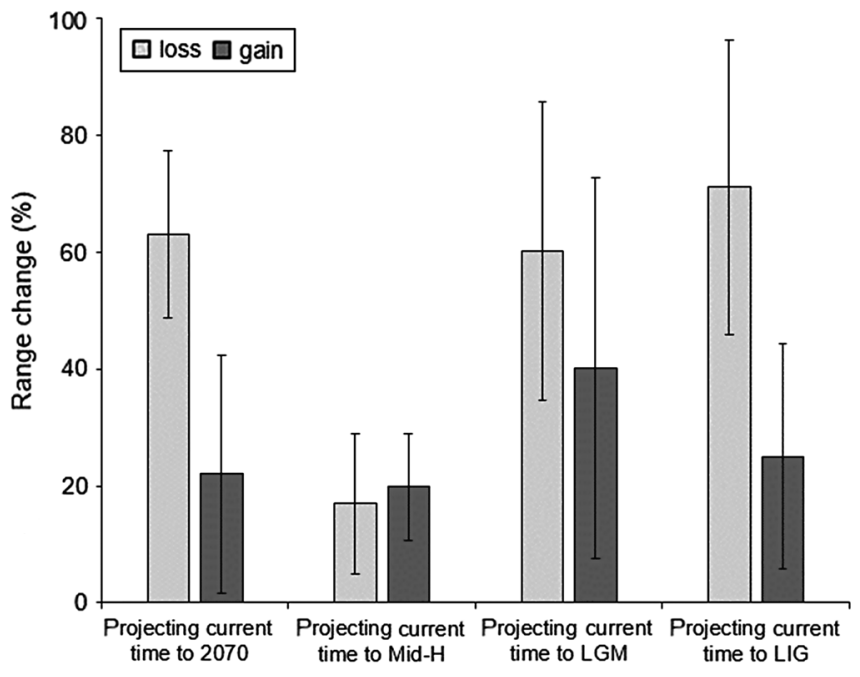

Figure 5. Comparison of the overall extent of habitat gain and loss for 60 koala-browse species between all modelled temporal windows and the present. Light grey and dark grey show the percentage of range loss and range gain, respectively, when comparing the present to the other time periods. Last Interglacial (LIG) $=\sim 128-116 \mathrm{ka}$, Last Glacial Maximum (LGM) $=\sim 23-19 \mathrm{ka}$, Mid-Holocene $(\mathrm{Mid}-\mathrm{H})=\sim 7-5 \mathrm{ka}$. Refer to Supplementary material Appendix 1 Table A5 for comparison of the extent of habitat gain and loss for 60 individual species between all modelled temporal windows and the present time. Also refer to overall gain and loss in mean suitabilities between time periods shown in Supplementary material Appendix 1 Fig. A4.

\section{Spatial distribution of koala fossils and date reliability}

Figure 2 illustrates the spatial distribution of existing koala fossil specimens and the reliability of the dates for each record. Koalas appear to have occupied the eastern and south-eastern regions (reliable dates for Naracoorte Caves only) of Australia continuously since at least $535 \pm 49 \mathrm{ka}$. In far south-western Australia, the oldest koala fossil specimen that could be reliably dated (category A) was $151 \pm 6 \mathrm{ka}$, and the youngest $70 \pm 4 \mathrm{ka}$ (Fig. 2). Additionally, there was a series from south-western Australia of possibly reliable-aged fossils (category B), with dates of $\sim 16$ ka for some Kangaroo Island specimens. Approximately one-third of the dates are unreliable (category C), so the timing of occupancy of the Nullarbor Plain is unresolved (Fig. 2).

\section{Discussion}

Our main objective was to assess the possible impacts of a varying climate on the distribution of koalas over the last 120 thousand years or so based on the hindcasted and projected niches of 60 of their main browse species. As expected, we revealed a highly variable response of individual tree species to the spatially explicit changes in climate conditions, but we were able to determine gross shifts in the likely extents of overall koala habitat during this interval (Fig. 4, Supplementary material Appendix 1 Fig. A2). By comparing the extent of past and present gains and losses of these 60 species, we found that overall gains exceeded losses between the Last Interglacial and the Last Glacial Maximum (i.e. koala habitats likely expanded during this interval), while all species had substantial losses of suitable habitat between the Last Glacial Maximum and the Mid-Holocene, and between the Mid-Holocene and the present (Fig. 4, Supplementary material Appendix 1 Fig. A2, Table A5). However, interpreting the projected differences between the Last Glacial Maximum and other time periods requires caution because of the much greater continental area and the absence of topsoil clay fraction data for the Last Glacial Maximum; these potentially overestimate the suitable niche space for each species. As such, our results support the hypothesis that extinctions of koala populations from the south-western and southern-central regions of Australia (as inferred from the fossil evidence) were likely a consequence of changing climates that restricted the distribution of their browse species over the last $\sim 20000$ years. The evidence provided by the fossil records suggest that koalas are particularly sensitive to climate change (see also McAlpine et al. 2015, Reckless et al. 2018), as is the case with specialist folivores in general (Black et al. 2014). The overall pattern appears to have been a substantial loss of habitat and an eastward retraction between the Last Glacial Maximum and the Mid-Holocene, as well as from the Mid-Holocene to the present (Fig. 4, Supplementary material Appendix 1 Fig. A2, Table A5).

Validating our projected eucalypt palaeodistributions individually using palaeo-vegetation data is challenging because of a lack of detailed palynological time series in Australia (the world's driest inhabited continent). However, we found several Eucalyptus palaeo-vegetation records in the projected areas of the tree species we modelled (Supplementary material Appendix 1 Fig. A5). Refer to Kershaw et al. (1991), Shulmeister (1992), D'Costa and Kershaw (1995), Harle et al. (1999) and Fletcher and Thomas (2007) for palynological time series and fossil pollen diagrams. We reasoned that variation in pollen concentrations of Eucalyptus in different locations of the study area could possibly arise from changes in climatic suitability for the 60 koala-browse species we projected. Comparing projected increases or decreases with the relevant time series of pollen records, we verified support for most of the trends we projected (Supplementary material Appendix 1 Fig. A5, Table A6).

Due to the poor taxonomic resolution of Eucalyptuslike pollen grains, we assume the modelled species to be representative of the wide ecological niche of Eucalyptus spp. Overall, our results are largely consistent with these Eucalyptus pollen records regarding the Last Glacial Maximum to the present in south-eastern Australia, and the Mid-Holocene to the present in south-western Tasmania. However, the decrease of suitability between the Mid-Holocene to the present in south-eastern Australia is not reflected in any palynological 
records. In south-western Tasmania, Eucalyptus densities slightly increased between the Mid-Holocene and the present in a fire-promoted moorland (Fletcher and Thomas 2007). This trend is consistent with our projections. In south-eastern Australia, pollen records from the Last Glacial Maximum indicate an open, herbaceous vegetation with lower Eucalyptus densities than in the Mid-Holocene and the present. This is mainly consistent with our projected trends presented above. Later, Eucalyptus densities increased and reached their current status during the Holocene (D’Costa and Kershaw 1995), even though our projection shows a moderate decrease of climate suitability from the Mid-Holocene to the present (refer to Supplementary material Appendix 1 Fig. A5).

We also determined that the rapid pace of climate change projected to 2070 under a realistic, high-emissions scenario is also likely to lead to large reductions in the climatically suitable habitats of koalas. Adams-Hosking et al. (2012) also predicted the contraction of koala habitats based on a single modelling framework (MaxEnt), concluding that the koala and its dietary species are likely to experience range contractions as climate change progresses, sometimes to regions outside the current distribution. This is of particular concern because the vulnerable conservation status of the species in most of its remaining range arises mainly from deforestation, disease, road kill and dog attacks (Beyer et al. 2018). While there could be some scope for retaining koalas in formerly unoccupied habitats, especially in South Australia (Sequeira et al. 2014), and possibly re-introducing them to long-unoccupied habitats in south-western Western Australia, or even new ones elsewhere, the outlook is not encouraging. Engaging in assisted colonization of koalas in response to climate change has been considered previously as a possible proactive strategy in koala conservation (Hoegh-Guldberg et al. 2008). However, the idea is controversial based on ecological, disease-risk and political issues (Loss et al. 2011, Waugh et al. 2016).

We assumed that the presence of koalas today is mainly attributable to the environmental variables that determine the presence of their main browse-tree species as suitable habitat (Seabrook et al. 2014). In reality, many eucalypt species have variable climatic tolerances and responses to climate change, such that some have the potential to persist beyond the extremities of the climate envelopes in which they currently thrive. In other words, we acknowledge that we have invoked an 'equilibrium assumption', which has its own particular problems (Varela et al. 2009, Václavík and Meentemeyer 2012, Saltré et al. 2013, 2015a, Booth 2017c). Fortunately, all of our four models produced results for each species that generally corresponded well with the known distribution of koalas today (Supplementary material Appendix 1) (Adams-Hosking et al. 2016). Given our set of modelled tree species was only considering their main food sources, it is possible that we did not fully capture the complexity of all koala habitats, especially given the species' need to have access to shelter trees to facilitate optimal thermoregulation (Briscoe et al. 2014, Crowther et al. 2014). Nonetheless, our goal was not to predict the total distribution of koalas in the past and future; rather, we aimed to quantify the changes in relative suitability of general habitat over time to identify the periods and regions of highest lability. However, we also modelled the currently suitable habitat of koalas on their present distribution and our results corresponded well with the mean suitability of the 60 koala-browse species (Supplementary material Appendix 1 Fig. A3). Our results showed that koala habitat quality in New South Wales has the highest suitability (mean suitability of all 60 koala-browse species for New South Wales $=0.84$ out of 1.0, and it was only 0.10 in Western Australia) (Supplementary material Appendix 1 Fig. A3).

Based on the high habitat suitability for some of the modelled tree species, we found suitable areas in the past and present in Tasmania in particular (Supplementary material Appendix 1 Fig. A2), despite no fossil evidence of koalas yet found there to confirm this possibility (Fig. 1). Indeed, during the Last Glacial Maximum in particular, the connection of Tasmania to the mainland and the suitability of habitat extending from currently occupied areas in the south-eastern part of the mainland support the biogeographical possibility of koalas having once occupied Tasmania. However, until fossil evidence is unearthed there, we cannot directly test this hypothesis.

Another potential complication with our models is the diet restrictions imposed by the peculiar physiology of a species that consumes eucalypts (refer to Moore et al. 2010, Law et al. 2017). Koala joeys remain in the mother's pouch for six to seven months post-parturition during which time they are nourished exclusively with the mother's milk (Martin and Handasyde 1999, Kalman and Levigne 2002). From between 22 and 30 weeks of age they also begin to feed on 'pap', which is a specific maternal derivation of koala faeces that facilitates the transition from milk to eucalypt leaves (Martin and Handasyde 1999). Pap contains gut bacteria essential for digesting eucalypt leaves, such that an abandoned koala joey denied pap has a limited chance of survival because it lacks the necessary gut microbes to digest its food (Kalman and Levigne 2002). While the plasticity of this herbivore-microbe-food plant specialization could conceivably accommodate the transition to different eucalypt species, it is plausible that the demise of some species, even when forests persist, might exacerbate the extinction risk of isolated populations.

We must also consider the potential biases imposed by the undersampling inherent in large databases of herbarium samples, even though the Australian eucalypts have been sampled comparatively well across $70 \%$ of the continent (GonzálezOrozco et al. 2014). There are also many inherent assumptions and limitations of presence-only data of this type, as well as with the modelling techniques projecting climate conditions and the envelopes themselves, such as scale and resolution mismatches (Wiens et al. 2009), dispersal barriers, lack of documentation on potential disturbances, and unknown or unforeseen biotic interactions (Elith et al. 2011). 
Regardless, the continental-scale projection of koala-browse species over multiple time periods does identify a useful baseline for both palaeo-biogeographical interpretations and possible conservation actions.

Given the suitability of areas like Tasmania, currently unoccupied regions of southern South Australia, and southwestern Western Australia, future conservation initiatives might debate the possibility of koala introductions in these areas as a means to reduce the extinction risk of the entire species. While controversial and potentially ecologically risky, our and others' work suggests that regions once suitable to koalas can become so again (Sequeira et al. 2014) if managed appropriately, potentially offsetting future habitat losses arising from climate change and human industry.

Acknowledgements - We thank J. Llewelyn for conceptual inputs. Funding - This research was done by the Australian Research Council Centre of Excellence for Australian Biodiversity and Heritage (CE170100015; EpicAustralia.org).

\section{References}

Adams-Hosking, C. et al. 2011a. Modelling climate-changeinduced shifts in the distribution of the koala. - Wildl. Res. 38: $122-130$.

Adams-Hosking, C. et al. 2011b. Modelling the potential range of the koala at the Last Glacial Maximum: future conservation implications. - Aust. Zool. 35: 983-990.

Adams-Hosking, C. et al. 2012. Modelling changes in the distribution of the critical food resources of a specialist folivore in response to climate change. - Divers. Distrib. 18: 847-860.

Adams-Hosking, C. et al. 2016. Use of expert knowledge to elicit population trends for the koala (Phascolarctos cinereus). - Divers. Distrib. 22: 249-262.

Ahmadi, M. et al. 2017. Combining landscape suitability and habitat connectivity to conserve the last surviving population of cheetah in Asia. - Divers. Distrib. 23: 592-603.

Allouche, O. et al. 2006. Assessing the accuracy of species distribution models: prevalence, kappa and the true skill statistic (TSS). - J. Appl. Ecol. 43: 1223-1232.

Anderson, B. J. et al. 2009. Using distribution models to test alternative hypotheses about a species' environmental limits and recovery prospects. - Biol. Conserv. 142: 488-499.

Araújo, M. B. and New, M. 2007. Ensemble forecasting of species distributions. - Trends Ecol. Evol. 22: 42-47.

Atlas of Living Australia 2018. - <www.ala.org.au> accessed 20 March 2018.

Austin, M. P. and Van Niel, K. 2011. Impact of landscape predictors on climate change modelling of species distributions: a case study with Eucalyptus fastigata in southern New South Wales, Australia. - J. Biogeogr. 38: 9-19.

Ayalew, L. and Yamagishi, H. 2005. The application of GIS-based logistic regression for landslide susceptibility mapping in the Kakuda-Yahiko Mountains, central Japan. - Geomorphology 65: 15-31.

Ayalew, L. et al. 2004. Landslide susceptibility mapping using GIS-based weighted linear combination, the case in Tsugawa area of Agano River, Niigata Prefecture, Japan. - Landslides 1: 73-81.

Bellard, C. et al. 2012. Impacts of climate change on the future of biodiversity. - Ecol. Lett. 15: 365-377.

Beyer, H. L. et al. 2018. Management of multiple threats achieves meaningful koala conservation outcomes. - J. Appl. Ecol. 55: 1966-1975.

Black, K. H. et al. 2014. Bearing up well? Understanding the past, present and future of Australia's koalas. - Gondwana Res. 25: 1186-1201.

Booth, T. H. 2013. Eucalypt plantations and climate change. - For. Ecol Manage. 301: 28-34.

Booth, T. H. 2017a. Impacts of climate change on eucalypt distributions in Australia: an examination of a recent study. - Aust. For. 80: 208-215.

Booth, T. H. 2017b. Assessing species climatic requirements beyond the realized niche: some lessons mainly from tree species distribution modelling. - Clim. Change 145: 259-271.

Booth, T. H. 2017c. Going nowhere fast: a review of seed dispersal in eucalypts. - Aust. J. Bot. 65: 401-410.

Booth, T. H. et al. 2014. BIOCLIM: the first species distribution modelling package, its early applications and relevance to most current MAXENT studies. - Divers. Distrib. 20: 1-9.

Booth, T. H. et al. 2015. Native forests and climate change: lessons from eucalypts. - For. Ecol. Manage. 347: 18-29.

Briscoe, N. J. et al. 2014. Tree-hugging koalas demonstrate a novel thermoregulatory mechanism for arboreal mammals. - Biol. Lett. 10: 20140235.

Bryan, B. A. 1997. A generic method for identifying regional koala habitat using GIS. - Geogr. Res. 35: 125-139.

Butt, N. et al. 2013. Eucalypts face increasing climate stress. - Ecol. Evol. 3: 5011-5022.

Cantor, S. B. et al. 1999. A comparison of C/B ratios from studies using receiver operating characteristic curve analysis. - J. Clin. Epidemiol. 52: 885-892.

Clark, P. U. et al. 2009. The Last Glacial Maximum. - Science 325: 710-714.

COHMAP Members 1988. Climatic changes of the last 18000 years: observations and model simulations. - Science 241: 1043-1052.

Crowther, M. S. et al. 2014. Climate-mediated habitat selection in an arboreal folivore. - Ecography 37: 336-343.

D'Costa, D. M. and Kershaw, A. P. 1995. A late Pleistocene and Holocene pollen record from Lake Terang, western plains of Victoria, Australia. - Palaeogeogr. Palaeoclimatol. Palaeoecol. 113: 57-67.

Davies, N. et al. 2014. Climate-driven changes in diet composition and physiological stress in an arboreal folivore at the semi-arid edge of its distribution. - Biol. Conserv. 172: 80-88.

Dormann, C. F. et al. 2007. Methods to account for spatial autocorrelation in the analysis of species distributional data: a review. - Ecography 30: 609-628.

Duursma, D. E. et al. 2013. Next-generation invaders? Hotspots for naturalised sleeper weeds in Australia under future climates. - PLoS One 8: e84222.

Elith, J. et al. 2010. The art of modelling range-shifting species. - Methods Ecol. Evol. 1: 330-342.

Elith, J. et al. 2011. A statistical explanation of MaxEnt for ecologists. - Divers. Distrib. 17: 43-57.

Ellis, W. et al. 2010. Climate change and the koala Phascolarctos cinereus: water and energy. - Aust. Zool. 35: 369-377. 
Fletcher, M. S. and Thomas, I. 2007. Holocene vegetation and climate change from near Lake Pedder, south-west Tasmania, Australia. - J. Biogeogr. 34: 665-677.

González-Orozco, C. E. et al. 2014. Biogeographical regions and phytogeography of the eucalypts. - Divers. Distrib. 20: 46-58.

Gordon, G. et al. 1988. A koala (Phascolarctos cinereus Goldfuss) population crash during drought and heatwave conditions in south-western Queensland. - Aust. Ecol. 13: 451-461.

Guillera-Arroita, G. et al. 2014. Maxent is not a presence-absence method: a comment on Thibaud et al. - Methods Ecol. Evol. 5: 1192-1197.

Guisan, A. et al. 1998. Predicting the potential distribution of plant species in an alpine environment. - J. Veg. Sci. 9: 65-74.

Guisan, A. et al. 2017. Habitat suitability and distribution models: with applications in R. - Cambridge Univ. Press.

Harle, K. J. et al. 1999. The contributions of uranium/thorium and marine palynology to the dating of the Lake Wangoom pollen record, western plains of Victoria, Australia. - Quat. Int. 57: $25-34$.

Hoegh-Guldberg, O. et al. 2008. Assisted colonization and rapid climate change. - Science 321: 345-346.

Hughes, L. 2003. Climate change and Australia: trends, projections and impacts. - Austral Ecol. 28: 423-443.

Hughes, L. et al. 1996. Climatic range sizes of Eucalyptus species in relation to future climate change. - Global Ecol. Biogeogr. Lett. 5: 23-29.

Jiménez-Valverde, A. 2014. Threshold-dependence as a desirable attribute for discrimination assessment: implications for the evaluation of species distribution models. - Biodivers. Conserv. 23: 369-385.

Kalman, B. and Levigne, H. 2002. The life cycle of a koala. - Crabtree Publishing Company.

Kershaw, A. P. et al. 1991. Palynological evidence for Quaternary vegetation and environments of mainland southeastern Australia. - Quat. Sci. Rev. 10: 391-404.

Kramer-Schadt, S. et al. 2013. The importance of correcting for sampling bias in MaxEnt species distribution models. - Divers. Distrib. 19: 1366-1379.

Law, B. et al. 2017. Development and field validation of a regional, management-scale habitat model: a koala Phascolarctos cinereus case study. - Ecol. Evol. 7: 7475-7489.

Lobo, J. M. et al. 2008. AUC: a misleading measure of the performance of predictive distribution models. - Global Ecol. Biogeogr. 17: 145-151.

Loss, S. R. et al. 2011. Assisted colonization: integrating conservation strategies in the face of climate change. - Biol. Conserv. 144: 92-100.

Lunney, D. et al. 2012. Koalas and climate change: a case study on the Liverpool Plains, north-west New South Wales. - In: Lunney, D. and Hutchings, P. (eds), Wildlife and climate change: towards robust conservation strategies for Australian fauna. Royal Zoological Society of NSW, Mosman, pp. $150-168$.

Lunney, D. et al. 2014. Extinction in Eden: identifying the role of climate change in the decline of the koala in south-eastern NSW. - Wildl. Res. 41: 22-34.

Lunney, D. et al. 2016. Interpreting patterns of population change in koalas from long-term datasets in Coffs Harbour on the north coast of New South Wales. - Aust. Mammol. 38: 29-43.

Martin, R. and Handasyde, K. A. 1999. The koala: natural history, conservation and management. - UNSW Press, Sydney, Australia.
Matusick, G. et al. 2013. Sudden forest canopy collapse corresponding with extreme drought and heat in a mediterranean-type eucalypt forest in southwestern Australia. - Eur. J. For. Res. 132: 497-510.

McAlpine, C. A. and Eyre, T. J. 2002. Testing landscape metrics as indicators of habitat loss and fragmentation in continuous eucalypt forests (Queensland, Australia). - Landscape Ecol. 17: 711-728.

McAlpine, C. A. et al. 2006. The importance of forest area and configuration relative to local habitat factors for conserving forest mammals: a case study of koalas in Queensland, Australia. - Biol. Conserv. 132: 153-165.

McAlpine, C. A. et al. 2015. Conserving koalas: a review of the contrasting regional trends, outlooks and policy challenges. - Biol. Conserv. 192: 226-236.

McGarigal, K. and McComb, W. 1995. Relationships between landscape structure and breeding birds in the Oregon Coast Range. - Ecol. Monogr. 65: 235-260.

Melzer, A. et al. 2000. Overview, critical assessment and conservation implications of koala distribution and abundance. - Conserv. Biol. 14: 619-628.

Mok, H. F. et al. 2012. Modelling the potential impact of climate variability and change on species regeneration potential in the temperate forests of south-eastern Australia. - Global Change Biol. 18: 1053-1072.

Moore, B. D. et al. 2010. Palatability mapping: a koala’s eye view of spatial variation in habitat quality. - Ecology 91: 3165-3176.

Nachtergaele, F. et al. 2009. Harmonized world soil database. - ISRIC, Wageningen.

Naimi, B. 2015. Usdm: uncertainty analysis for species distribution models. - R package ver. 1.1-12.

Nenzén, H. K. and Araújo, M. B. 2011. Choice of threshold alters projections of species range shifts under climate change. - Ecol. Model. 222: 3346-3354.

NSW Dept of Planning and Environment 2016. Explanation of intended effect: state environmental planning policy no. 44 - koala habitat protection. - 4-13, Sydney, Australia.

Papadopoulou-Vrynioti, K. et al. 2013. Karst collapse susceptibility mapping considering peak ground acceleration in a rapidly growing urban area. - Eng. Geol. 158: 77-88.

Parmesan, C. 2006. Ecological and evolutionary responses to recent climate change. - Annu. Rev. Ecol. Evol. Syst. 37: 637-669.

Parmesan, C. and Yohe, G. 2003. A globally coherent fingerprint of climate change impacts across natural systems. - Nature 421: 37-42.

Pearson, G. A. et al. 2009. Frayed at the edges: selective pressure and adaptive response to abiotic stressors are mismatched in low diversity edge populations. - J. Ecol. 97: 450-462.

Pearson, R. G. et al. 2002. SPECIES: a spatial evaluation of climate impact on the envelope of species. - Ecol. Model. 154: 289-300.

Phillips, S. et al. 2000. The tree species preferences of koalas (Phascolarctos cinereus) inhabiting forest and woodland communities on Quaternary deposits in the Port Stephens area, New South Wales. - Wildl. Res. 27: 1-10.

Reckless, H. J. et al. 2018. A review of climatic change as a determinant of the viability of koala populations. - Wildl. Res. 44: 458-470.

Renner, I. W. and Warton, D. I. 2013. Equivalence of MAXENT and Poisson point process models for species distribution modeling in ecology. - Biometrics 69: 274-281. 
Rodríguez-Rey, M. et al. 2015. Criteria for assessing the quality of Middle Pleistocene to Holocene vertebrate fossil ages. - Quat. Geochronol. 30: 69-79.

Rodríguez-Rey, M. et al. 2016. A comprehensive database of quality-rated fossil ages for Sahul's Quaternary vertebrates. - Sci. Data 3: 160053.

Saltré, F. et al. 2013. Climate or migration: what limited European beech post-glacial colonization? - Global Ecol. Biogeogr. 22: 1217-1227.

Saltré, F. et al. 2015a. Uncertainties in dating constrain model choice for inferring extinction time from fossil records. - Quat. Sci. Rev. 112: 128-137.

Saltré, F. et al. 2015b. How climate, migration ability and habitat fragmentation affect the projected future distribution of European beech. - Global Change Biol. 21: 897-910.

Saltré, F. et al. 2016. Climate change not to blame for late Quaternary megafauna extinctions in Australia. - Nat. Commun. 7: 10511.

Santika, T. et al. 2014. Modelling species distributional shifts across broad spatial extents by linking dynamic occupancy models with public-based surveys. - Divers. Distrib. 20: 786-796.

Schepen, A. and Wang, Q. J. 2013. Toward accurate and reliable forecasts of Australian seasonal rainfall by calibrating and merging multiple coupled GCMS. - Mon. Weather Rev. 141: 4554-4563.

Seabrook, L. et al. 2014. Determining range edges: habitat quality, climate or climate extremes? - Divers. Distrib. 20: 95-106.

Sequeira, A. M. M. et al. 2012. Ocean-scale prediction of whale shark distribution. - Divers. Distrib. 18: 504-518.

Sequeira, A. M. M. et al. 2014. Distribution models for koalas in South Australia using citizen science-collected data. - Ecol. Evol. 4: 2103-2114.

Shabani, F. et al. 2016. A comparison of absolute performance of different correlative and mechanistic species distribution models in an independent area. - Ecol. Evol. 6: 5973-5986.

Shabani, F. et al. 2017. Climate modelling shows increased risk to Eucalyptus sideroxylon on the eastern coast of Australia compared to Eucalyptus albens. - Plants 6: 58.

Shabani, F. et al. 2018. A comparative modeling study on non-climatic and climatic risk assessment on Asian tiger mosquito (Aedes albopictus). - PeerJ 6: e4474.

Shabani, F. et al. 2019. Data from: Climate-driven shifts in the distribution of koala-browse species from the Last Interglacial to the near future. - Dryad Digital Repository, <https://doi. org/10.5061/dryad.r79k7sc $>$.

Shulmeister, J. 1992. A Holocene pollen record from lowland tropical Australia. - Holocene 2: 107-116.

Supplementary material (available online as Appendix ecog04530 at <www.ecography.org/appendix/ecog-04530>). Appendix 1.
Steig, E. J. 1999. Mid-Holocene climate change. - Science 286: $1485-1487$.

Stirling, C. H. et al. 1998. Timing and duration of the Last Interglacial: evidence for a restricted interval of widespread coral reef growth. - Earth Planet. Sci. Lett. 160: 745-762.

Strona, G. and Bradshaw, C. J. A. 2018. Co-extinctions annihilate planetary life during extreme environmental change. - Sci. Rep. 8: 16724.

Svenning, J. C. et al. 2011. Applications of species distribution modeling to paleobiology. - Quat. Sci. Rev. 30: 2930-2947.

Swets, J. A. 1988. Measuring the accuracy of diagnostic systems. - Science 240: 1285-1293.

Tehrany, M. S. et al. 2017. Soil erosion susceptibility mapping for current and 2100 climate conditions using evidential belief function and frequency ratio. - Geomat. Nat. Haz. Risk. 8: $1695-1714$.

Thomas, C. 2010. Climate, climate change and range boundaries. - Divers. Distrib. 16: 488-495.

Thuiller, W. et al. 2008. Predicting global change impacts on plant species' distributions: future challenges. - Perspect. Plant Ecol. Syst. 9: 137-152.

Thuiller, W. et al. 2009. BIOMOD - a platform for ensemble forecasting of species distributions. - Ecography 32: 369-373.

Urban, M. C. 2015. Accelerating extinction risk from climate change. - Science 348: 571.

Václavík, T. and Meentemeyer, R. 2012. Equilibrium or not? Modelling potential distribution of invasive species in different stages of invasion. - Divers. Distrib. 18: 73-83.

Varela, S. et al. 2009. Is current climatic equilibrium a guarantee for the transferability of distribution model predictions? A case study of the spotted hyena. - J. Biogeogr. 36: 1645-1655.

Watanabe, S. et al. 2011. MIROC-ESM 2010: model description and basic results of CMIP5-20c3m experiments. - Geosci. Model Dev. 4: 845-872.

Waugh, C. et al. 2016. Koala translocations and Chlamydia: managing risk in the effort to conserve native species. - Biol. Conserv. 197: 247-253.

Wiens, J. A. et al. 2009. Niches, models and climate change: assessing the assumptions and uncertainties. - Proc. Natl Acad. Sci. USA 106: 19729-19736.

Wisz, M. S. et al. 2008. Effects of sample size on the performance of species distribution models. - Divers. Distrib. 14: 763-773.

Wu, H. et al. 2012. The dietary preferences of koalas, Phascolarctos cinereus, in southwest Queensland. - Aust. Zool. 36: 93-102.

Xiao, J. et al. 2006. Evaluating urban expansion and land use change in Shijiazhuang, China, by using GIS and remote sensing. - Landscape Urban Plan. 75: 69-80. 